\title{
HIGH FREQUENCY ZNO-ON-DIAMOND MONOLITHIC FILTERS
}

\author{
R. Abdolvand ${ }^{1}$ and F. Ayazi ${ }^{2}$
}

${ }^{1}$ School of Electrical and Computer Engineering, Oklahoma State University, Stillwater, Oklahoma, USA

${ }^{2}$ School of Electrical and Computer Engineering, Georgia Institute of Technology, Atlanta, Georgia, USA

\section{ABSTRACT}

This paper reports on the design and implementation of acoustically-coupled thin-film ZnO-on-diamond resonators fabricated on a single resonant structure (monolithic filter). These small devices benefit from the large elastic modulus of diamond (largest in nature) to reach higher center frequencies while the frequency-determining dimension of the device stays in a range comfortably feasible to fabricate using conventional photolithography and etching tools. Lateral mode filters with center frequencies up to $900 \mathrm{MHz}$ are demonstrated with $1.4 \mathrm{x}$ frequency increase compared to the devices made on silicon.

\section{INTRODUCTION}

In a monolithic acoustic filter multiple modes of a single resonant structure are coupled in order to achieve a higher order system. The same technique has been implemented in monolithic crystal filter (MCF) technology for decades [1]. The majority of the MCF's have been designed based on coupled thickness modes of a quartz plate [1,2]. With the emergence of thin-film technologies very high frequency (few $\mathrm{GHz}$ ) piezoelectric filters are demonstrated and commercialized (e.g. film bulk acoustic resonator filters). However, the technique most attractive for lowfrequency crystal filters (monolithic mode coupling) have not been yet adopted for these new-generation of acoustic devices. Lateral and thickness mode monolithic thin-film piezoelectric-on-silicon (TPoS) filters were demonstrated for the first time in [3]. In this work, we use a nanocrystalline diamond (NCD) film as the resonant structure in place of the silicon to achieve higher center frequencies.

\section{FILTER DESIGN}

A monolithic thin-film piezoelectric-on-diamond (TPoD) filter is structurally the same as a two-port TPoD resonator (Fig. 1) [4]. The difference between a TPoD resonator and a filter is mainly in the top electrode pattern. In a filter configuration the top electrode is properly patterned to enable excitation of the dual resonance modes of a released structure. These two modes include a symmetric mode in which the displacement under both electrodes is in-phase, and an asymmetric mode in which the developed strain field under the two electrodes is $180^{\circ}$ out of phase.

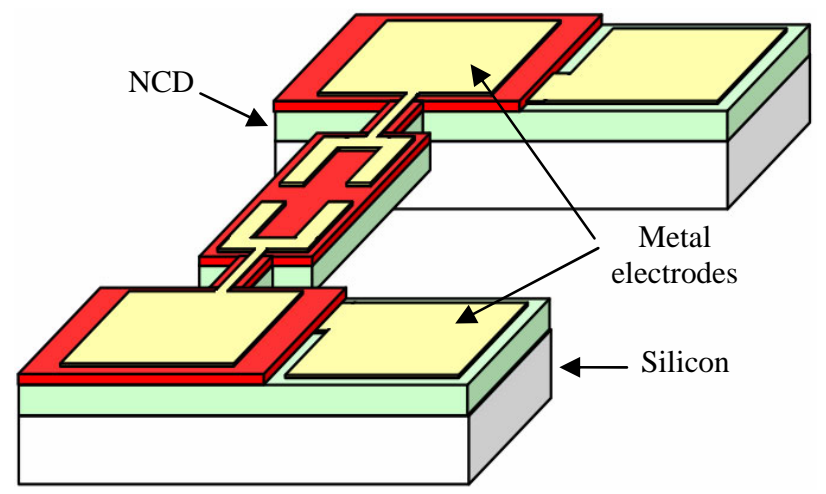

Figure 1: The schematic diagram of a piezoelectric-on-diamond monolithic filter.
In Fig. 2 the dual third harmonic lateral extensional modeshapes that can be excited by the electrode pattern shown in Fig. 1 are simulated using the COMSOL package.

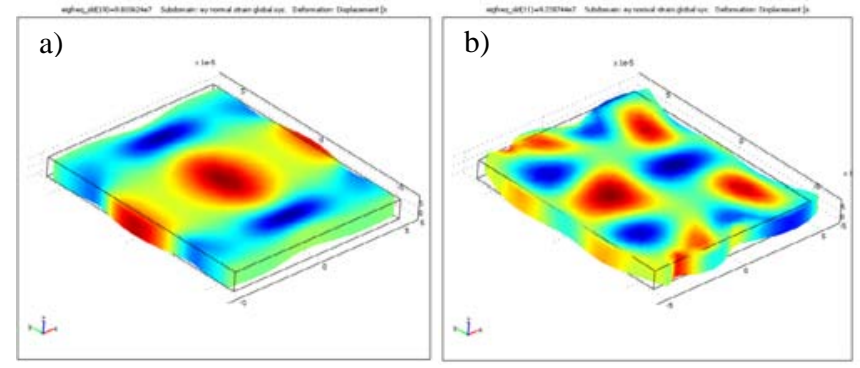

Figure 2: The simulated dual third harmonic lateral extensional mode-shapes which are coupled in the filter shown in Fig. 1; a) inphase and b) out-of-phase.

Theoretically, all classes of resonance modes including flexural and extensional can be employed in a monolithic filter. In this work, a flexural-mode as well as a high-frequency (RF) lateral-extensional mode $\mathrm{ZnO}$-on-diamond filter is presented. For the flexural-mode filter, the resonant structure is a long beam. The top electrode in this device is a metal strip which is split in half on top of the $\mathrm{ZnO}$ layer. The simulated dual flexural mode-shapes excited in this filter are presented in Fig. 3.

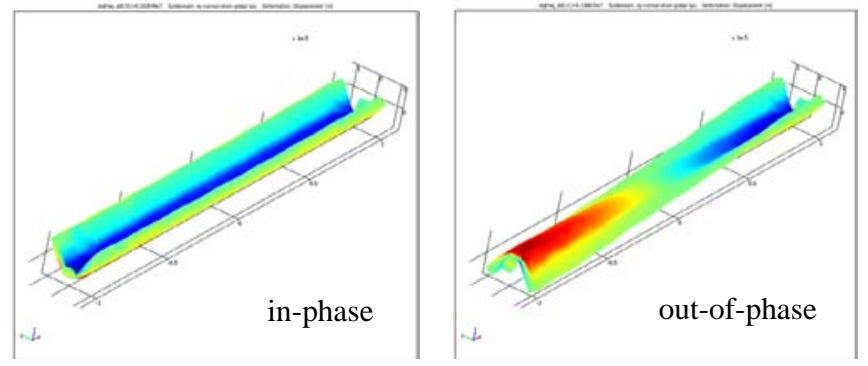

Figure 3: The simulated dual width flexural mode-shapes of a long beam.

On the other hand, the extensional mode filter consists of a wide rectangular membrane with a plurality of metal fingers on top of the $\mathrm{ZnO}$ layer. These fingers are designed to match the strain field pattern in a high-order lateral-extensional mode-shape of the structure (extension of the device shown in Fig. 1 in width direction).

\section{EXPERIMENTAL RESULTS}

The process flow for fabrication of $\mathrm{ZnO}$-on-diamond filters is schematically presented in Fig. 4. The resonant structure consists of a stack of $\mathrm{ZnO}$ on NCD. However, an optional thin layer of polished silicon dioxide can be included in the structure. The starting substrate is a silicon wafer with $\sim 2 \mu \mathrm{m}$ of NCD deposited on top. The surface roughness of the diamond crucially affects the quality of the sputter-deposited piezoelectric layer. Therefore, the optional intermediate oxide layer is used to act as a buffer layer to compensate for the roughness of the diamond after polishing [4]. It also performs as a passive temperature compensation mechanism. 


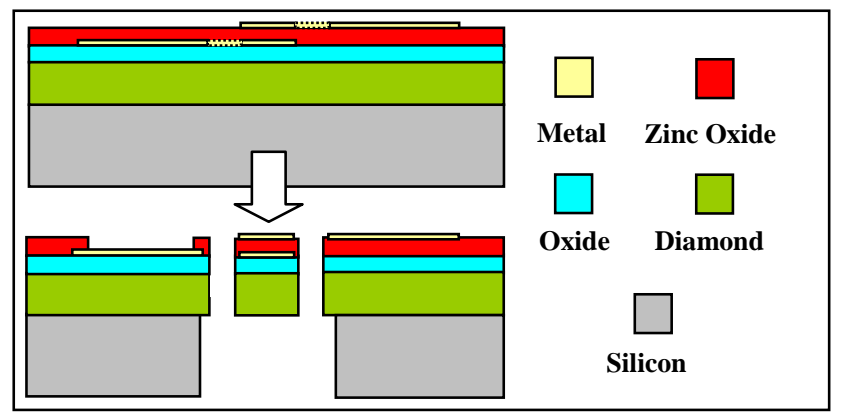

Figure 4: The schematic of the process flow

After depositing all the layers on the substrate, the access hole to the bottom electrode is etched in $\mathrm{ZnO}$. Then, the resonant structure is defined by etching the stack from the top and is released by etching the silicon from the backside.

Fabricated devices are characterized on a high frequency probe station using GSG probes. The proper calibration procedure is performed before each measurement. The frequency response of Fig. 5 is measured from the flexural-mode filter shown in the inset SEM picture. The device is a $20 \mu \mathrm{m}$ wide, $400 \mu \mathrm{m}$ long beam. The measured high out-of-band rejection (-50dB@78MHz) is due to the electrical isolation between the input and output ports which is a signature characteristic of acoustically-coupled filters. The insertion loss and the pass-band ripple of the filter are improved by terminating the filter with $500 \Omega$ loads.

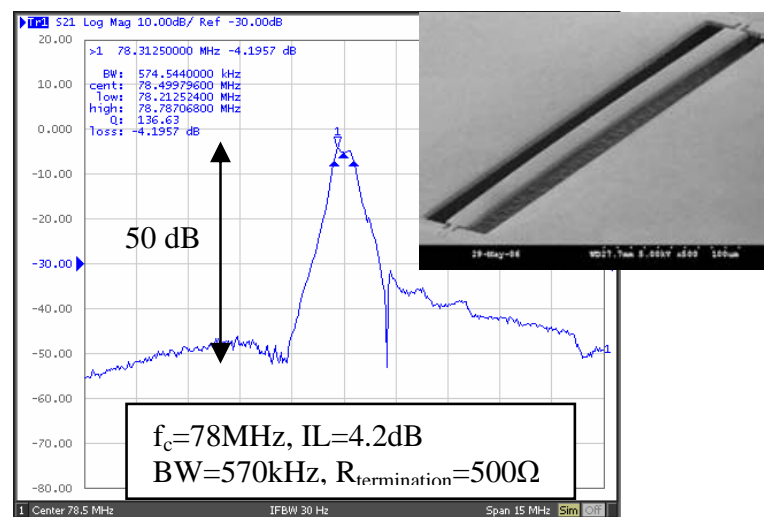

Figure 5: The frequency response of the flexural Zno-onnanocrystalline diamond monolithic filter

In this flexural mode device a thin layer of silicon dioxide $(0.5 \mu \mathrm{m})$ was deposited and polished to promote the growth of cplane $\mathrm{ZnO}$ grains on the rather rough surface of the NCD film. The temperature coefficient of frequency in this device was measured to be less than $8 \mathrm{ppm} /{ }^{\circ} \mathrm{C}$ as a result of including the oxide film in the structure. By modifying the deposition parameters, the grain size in the NCD film can be controlled. Smaller grain size NCD layer translates into a smoother surface which may eliminate the need for a polished intermediate layer.

Frequency responses of two lateral-extensional filters with identical layout (Fig. 6) fabricated on different substrates are compared in Fig. 7. The device fabricated on a $4 \mu \mathrm{m}$ SOI substrate has a center frequency of $640 \mathrm{MHz}(\mathrm{BW} 1.5 \mathrm{MHz})$ where as the same device made on the $2 \mu \mathrm{m}$ thick NCD film shows a $1.4 \mathrm{x}$ increase in the center frequency $(\sim 900 \mathrm{MHz})$ with a $3 \mathrm{MHz}$ bandwidth. The ZnO is directly deposited on the NCD film with less than $10 \mathrm{~nm}$ surface roughness. Relatively high insertion loss of the filter can be partially attributed to the lower coupling coefficient of the piezoelectric film.

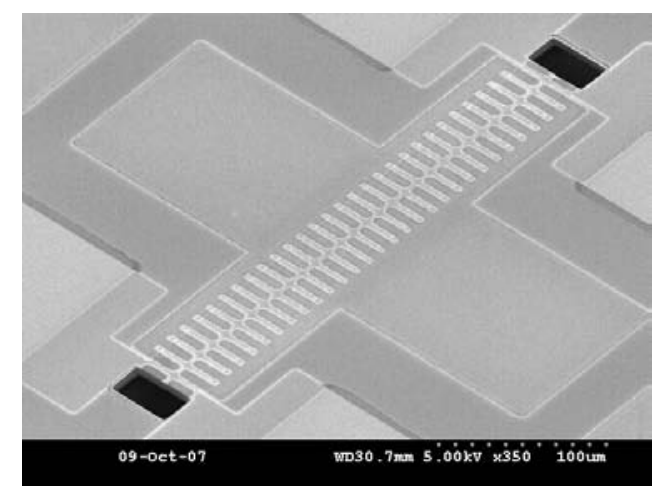

Figure 6: The SEM picture of the lateral-mode monolithic filter. The center-to-center finger pitch is $10 \mu \mathrm{m}$ in this device.
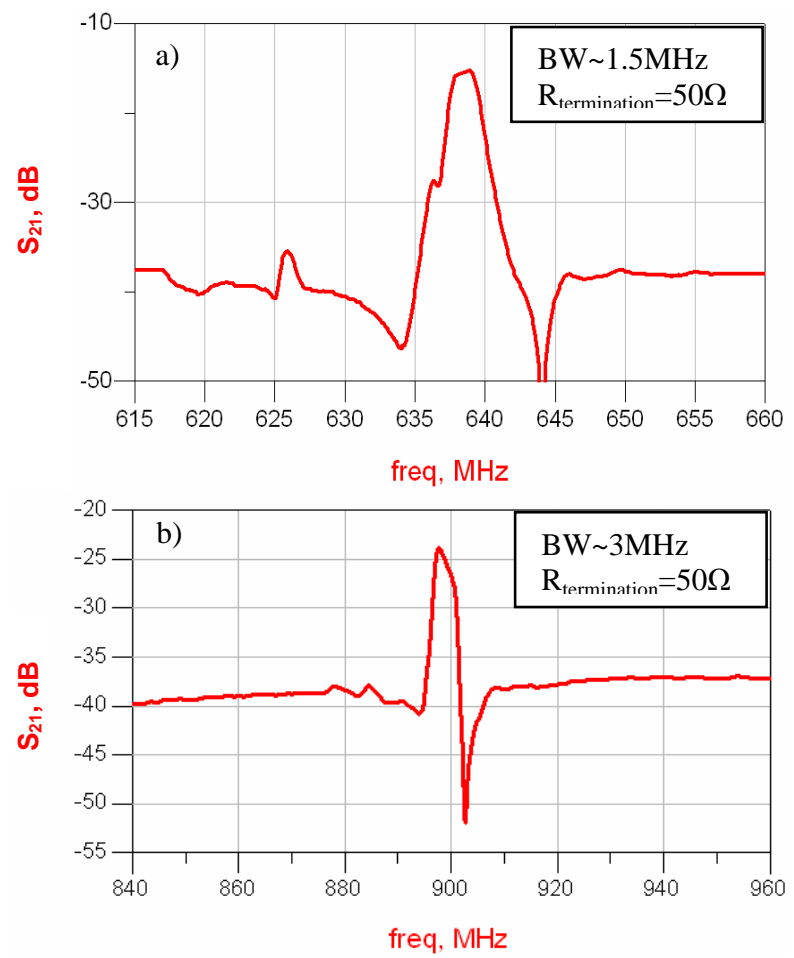

Figure 7: Measured frequency response plots of the device shown in Fig.6 fabricated on; a)SOI and b)Nanocrystalline diamond.

\section{ACKNOWLEDGEMENT}

Authors wish to thank Advanced Diamond Technology for providing the nanocrystalline diamond films.

\section{REFERENCES}

[1] W. D. Beaver, "Theory and design of the monolithic crystal filter,” 21st Annual Symposium on Frequency Control, pp. 179-199, 1967.

[2] R. G. Kinsman, “A history of crystal filters,” Proceedings of the 1998 IEEE International Frequency Control Symposium, May 1998, pp.563-570.

[3] R. Abdolvand, F. Ayazi, "Monolithic thin-film piezoelectricon-substrate filters" Proc. IEEE MTT-S International Microwave Symposium (IMS 2007), June 2007, pp. 509-512.

[4] R. Abdolvand, G. K. Ho, J. Butler, F. Ayazi, "ZnO-onnanocrystalline-diamond lateral bulk acoustic resonators," Proc. of IEEE Micro Electro Mechanical Systems Conference, Jan. 2007, pp. 795-798. 